\begin{tabular}{|c|l|}
\hline Title & Quantification of integral data effectiveness using the concept of active subspace in evaluated nuclear data validation \\
\hline Author(s) & Chiba, Go; Imazato, Daichi \\
\hline Citation & $\begin{array}{l}\text { Journal of nuclear science and technology, 57(11), 1245-1255 } \\
\text { https://doi.org/10.1080/00223131.2020.1780992 }\end{array}$ \\
\hline Issue Date & 2020-11 \\
\hline Doc URL & http://hdl.handle.net/2115/83118 \\
\hline Rights & $\begin{array}{l}\text { This is an A ccepted Manuscript of an article published by Taylor \& Francis in Journal of nuclear science and } \\
\text { technology on Nov 2020, available online: http:/Www.tandfonline.com/10.1080/00223131.2020.1780992. }\end{array}$ \\
\hline Type & article (author version) \\
\hline File Information & paper.pdf \\
\hline
\end{tabular}

Instructions for use 


\title{
Quantification of integral data effectiveness using the concept of active subspace in evaluated nuclear data validation
}

\author{
Go Chiba $^{1 *}$, Daichi Imazato ${ }^{1}$ \\ ${ }^{1}$ Division of applied quantum science and engineering, faculty of engineering, Hokkaido University, Kita \\ 13 Nishi 8, Kita-ku, Sapporo 060-8628, Japan
}

In order to know performance of evaluated nuclear data in reactor physics or radiation shielding calculations, its benchmark testing with integral data is mandatory. Nowadays we have a huge amount of integral data, but some of them are quite similar with each other. We need to know independency of available integral data, and also to choose a proper set of a limited number of integral data for benchmark calculations. Furthermore, it is beneficial to know how effective a set of integral data is for independent validation of each nuclear data in performing validation test of nuclear data. In order to quantify the effectiveness of integral data in nuclear data validation, we propose several methods based on a concept of the active subspace. With the proposed methods, we can quantify independency of a set of integral data, choose a minimum set of proper integral data, and quantify possibility of independent validation of nuclear data from a set of integral data. These methods are adopted to a set of fictitious integral data and a set of actual integral data including experimental data about $k_{\text {eff }}$ and reaction rate ratio. Through these applications, effectiveness of these integral data has been succesfully quantified. Furthermore, the proposed concept is utilized to interpret the nuclear data compensation effect, which has been recently discussed in the community of nuclear data. 
Keywords: evaluated nuclear data, integral data, benchmark calculations, active subspace 


\section{Introduction}

When one solves reactor physics or radiation shielding problems, fundamental data describing interaction of neutrons with nucleus are essential, and those are well known as nuclear data in the field of nuclear and radiation engineering. True values of nuclear data should exist, but those cannot be theoretically determined. Thus, evaluation process of nuclear data using information of relevant measurable data and nuclear physics models is required. These evaluated nuclear data are provided to users who have interests on reactor physics or radiation shielding problems. A huge amount of experimental data related to reactor physics and radiation shielding have been obtained at various facilities in the world. Those data are referred to as integral data in the community of nuclear data since parameters of integral data, integral parameters, are complicatedly dependent on many different nuclear data. Those integral data can be utilized to quantify performance of evaluated nuclear data, and many integral data with detail information required for evaluated nuclear data validation have been collected through international projects such as ICSBEP[1].

As described above, the number of available integral data has become enormous now, so it is important to choose a proper set of integral data when testing evaluated nuclear data with them. When the number of concerned nuclear data is limited, a relatively small number of important integral data sensitive to these concerned nuclear data can be chosen, and different evaluated nuclear data libraries are tested against this set of integral data[2]. When a new evaluated nuclear data library is developed, benchmark calculations of this library should be conducted comprehensively, and in such cases a wide variety of integral data should be chosen. In the case of Japanese general-purpose evaluated nuclear data library JENDL-4.0[3], a set of important integral data are chosen by experts[4]. In order to choose a proper set of integral data, similarity and dependency among integral data should be carefully examined. Generally this kind of work has been conducted by experts 
based on their experiences and knowledge, but it is preferable that a procedure how to choose a proper set of integral data is established.

When a set of integral data is given, it is impossible to determine a unique set of nuclear data which exactly reproduces these integral data since generally the number of integral data is much smaller than the number of nuclear data. However, by using combinations of several integral data which contribute differently to nuclear data, it would not be impossible to determine a proper value of some of nuclear data. To know how effective a set of integral data is for independent validation of each nuclear data is beneficial in performing validation test of nuclear data against integral data. To this purpose, a method to quantify possibility of validation of each nuclear data with a given set of integral data is desired to be developed.

In the present paper, we propose to adopt a concept of the active subspace, which has been extensively studied in the field of nuclear engineering recently[5], to these subjects. To quantify effectiveness of integral data available for us to test evaluated nuclear data, we propose methods to quantify independency of a set of integral data, to choose a minimum set of proper integral data from a huge amount of integral data, and to quantify possibility of validation of each nuclear data with a given set of integral data. Furthermore, this concept is utilized to interpret the nuclear data compensation effect[6], which has been recently raised in the community of nuclear data.

In Section 2, the above-mentioned procedures with the basic concept are described. Numerical methods and tools used to test the proposed procedures are explained in Section 3 , and the proposed methods are applied to a set of fictitious integral data and a set of actual integral data in Section 4. Application of the proposed methods to interpret the nuclear data compensation effect is also described in this section. Section 5 is devoted to conclude the present study and to provide future perspective. 


\section{Concept and procedure}

\subsection{Basic concept}

Within multi-energy group representation generally adopted in deterministic calculations for reactor physics and radiation shielding, nuclear data which are dependent on incident/emitted particle energy, such as reaction cross sections, are discretized with energy groups. We regard each energy group-dependent nuclear data as a vector, and assume that each nuclear data vector is orthogonal to other nuclear data vectors. From a set of these nuclear data vectors, a nuclear data space can be defined. Dimension of a nuclear data space is equal to the number of nuclear data.

Relationship between nuclear data and integral data can be quantified by a sensitivity of an integral parameter with respect to each nuclear data. A sensitivity of an integral parameter $p$ to nuclear data $\sigma, s$, is generally defined as

$$
s=\frac{\partial p}{\partial \sigma} \cdot \frac{\sigma}{p}
$$

and this definition is used in the present study. Since a sensitivity of one integral parameter is defined to all the nuclear data, a sensitivity can be regarded as a vector in the nuclear data space, and a set of sensitivity vectors can span a subspace of the nuclear data space. We call this subspace an integral data space. Dimension of an integral data space is equal to or smaller than the number of sensitivity vectors, i.e. the number of integral data. If similarity among a set of integral data is large, dimension of an integral data space becomes small.

We have to remember that each integral data should include uncertainty coming from its measurement: experimental uncertainty. Integral data with small experimental uncertainties should have higher priority than integral data with large experimental uncertainties. Also, even though there are some integral data whose sensitivity profiles are very close to each other, use of all these information would be beneficial if experimental uncertainties of these integral data are large and independent with each other. In the present 
paper, we will not care about these issues about experimental uncertainties.

\subsection{Quantification of independency among integral data}

As mentioned in the introduction, there have been a large number of integral data accumulated in the past, but some of them are quite similar with each other because of similarity in loaded nuclear fuels, used structural materials, geometrical specifications, etc. Similarity among integral data can be quantified by using sensitivities of integral data with respect to nuclear data, and independency of a set of integral data can be quantified as a dimension of an integral data space described in the preceding subsection.

Vectors spanning an integral data space can be easily calculated by mathematical procedures, and we utilize the singular value decomposition (SVD) technique as follows. Here we consider a sensitivity matrix $\mathbf{S}_{I \times J}=\left(\mathbf{s}_{1} \mathbf{s}_{2} \cdots \mathbf{s}_{J}\right)$ where $\mathbf{s}_{j}$ is a sensitivity vector of the $j$ th integral parameter and is defined as $\mathbf{s}_{j}=\left(\frac{d p_{j}}{d \sigma_{1}} \cdot \frac{\sigma_{1}}{p_{j}} \frac{d p_{j}}{d \sigma_{2}} \cdot \frac{\sigma_{2}}{p_{j}} \cdots \frac{d p_{j}}{d \sigma_{I}} \cdot \frac{\sigma_{I}}{p_{j}}\right)^{T}$, where $p_{j}$ is the $j$ th integral parameter and $\sigma_{i}$ is the $i$ th nuclear data. The superscript $T$ is for vector and matrix transposition. The numbers of nuclear data and integral parameters are denoted to as $I$ and $J$. To obtain a spanning set of the integral data space, SVD of the matrix $\mathbf{S}$ is carried out as $\mathbf{S}=\mathbf{U D V}^{T}$ where $\mathbf{U}_{I \times I}=\left(\mathbf{u}_{1} \mathbf{u}_{2} \cdots \mathbf{u}_{L} \cdots \mathbf{u}_{I}\right), \mathbf{V}_{J \times J}=$ $\left(\mathbf{v}_{1} \mathbf{v}_{2} \cdots \mathbf{v}_{L} \cdots \mathbf{v}_{J}\right)$ and

$$
\mathbf{D}_{I \times J}=\left(\begin{array}{ccccccc}
\Sigma_{1}^{2} & 0 & \cdots & 0 & 0 & \cdots & 0 \\
0 & \Sigma_{2}^{2} & \cdots & 0 & 0 & \cdots & 0 \\
\vdots & \vdots & \ddots & 0 & 0 & \cdots & 0 \\
0 & 0 & 0 & \Sigma_{L}^{2} & 0 & \cdots & 0 \\
0 & 0 & 0 & 0 & 0 & \cdots & 0 \\
\vdots & \vdots & \vdots & \vdots & \vdots & \ddots & 0 \\
0 & 0 & 0 & 0 & 0 & \cdots & 0
\end{array}\right)
$$

where $L$ and $\mathbf{u}_{i}(1 \leq i \leq L)$ are dimension and orthonormal basis vectors of an integral data space considered here. Note that singular values $\Sigma_{i}$ are arranged in descending order in the present paper.

Generally, the same number of singular values as the number of integral parameters or 
nuclear data is numerically calculated, so we need to determine a threshold value for singular values to separate non-negligible components from noise (meaningless) components. In the present work, we propose the following procedure to determine effective dimension of an integral data space:

(1) A set of orthonormal basis vectors of an integral data space is obtained by SVD of a sensitivity matrix. Set $n=1$.

(2) Choose $n$ principal basis vectors corresponding to the largest singular values, and construct a $n$-dimensional subspace spanned by these $n$ basis vectors.

(3) Each of sensitivity vectors is orthogonally projected to this subspace, and norms of the projected vectors are calculated. Note that sensitivity vectors are normalized so as to make their norms unity in this step.

(4) If the minimum value of the norms obtained at the step 3 is smaller than criteria, $n$ is increased by one, and a procedure from the step 2 is carried out again. If the minimum norm is larger than the criteria, this procedure is terminated and the dimension of the integral data space is determined as $n$.

This procedure checks reproducibility of each sensitivity vector by the constructed subspace, and it is based on rather a physical aspect than a mathematical aspect which simply uses criteria on singular values. Setting of the criteria of this reproducibility is important, and it should be strongly related to accuracy and reliability of sensitivity vectors.

\subsection{A method choosing minimum data set}

There are a huge amount of available integral data, but it is unrealistic to use all of them when testing evaluated nuclear data. Here we propose a method to choose a minimum data set from integral data based on the proposed concept. The procedure is as follows:

(1) The first principal basis vector of the integral data space having the largest singular 
value is taken, and inner products of this vector and each normalized sensitivity vector are calculated. After doing this, a sensitivity vector which gives the largest inner product is chosen as the first integral data.

(2) Construct a subspace spanned by sensitivity vectors of the chosen integral data.

(3) Each of non-chosen normalized sensitivity vectors is orthogonally projected to the subspace constructed at the step 2. If norms of all these projected vectors are larger than criteria, this procedure is terminated. If not, a sensitivity vector which gives the minimum norm is additionally chosen, and go back to the step 2 .

Criteria at the step 3 are arbitrary, so users should set it.

\subsection{Quantification of nuclear data independent validation possibility with integral data}

Generally a proper value of nuclear data cannot be uniquely determined from integral data since each integral data is sensitive to various nuclear data. Combined use of a set of integral data, however, would make it possible to validate some of nuclear data independently. This can be interpreted by using the concept of the active subspace as follows. If a nuclear data vector exists on an integral data space, this means that a fictitious sensitivity vector which is sensitive only to this nuclear data can be generated by a combined use of these integral data, and it can be said that this nuclear data can be independently validated with this set of the integral data. On the other hand, if a nuclear data vector does not exist on the integral data space, it means that it is impossible to independently validate this nuclear data with this set of the integral data. Here we propose a method to quantify a possibility of independent validation of each nuclear data using a norm of an orthogonally-projected vector of a nuclear data vector to the integral data space. This concept is illustrated in Fig. 1 where a two-dimensional integral data space and a nuclear data vector are presented. 
[Figure 1 about here.]

Orthogonally projection of a nuclear data vector to an integral data space can be carried out as follows. Let us consider a vector corresponding to the $i$ th nuclear data, and it can be represented as $\mathbf{e}_{i}$ in the nuclear data space. The vector $\mathbf{e}_{i}$ is a unit vector whose $i$ th entry is unity and others are zero. An orthgonally-projected vector of $\mathbf{e}_{i}$ to the integral data space, $\mathbf{e}_{i}^{\prime}$, can be written as

$$
\mathbf{e}_{i}^{\prime}=\overline{\mathbf{U}} \overline{\mathbf{U}}^{T} \mathbf{e}_{i}
$$

where $\overline{\mathbf{U}}$ is defined as

$$
\overline{\mathbf{U}}_{L \times L}=\left(\mathbf{u}_{1} \mathbf{u}_{2} \cdots \mathbf{u}_{L}\right)
$$

Let us be reminded that $\mathbf{u}_{l}$ is the $l$ th basis vector of the integral data space. The possibility of independent validation of the $i$ th nuclear data is quantified as the Euclidean norm of $\mathbf{e}_{i}^{\prime}:\left\|\mathbf{e}_{i}^{\prime}\right\|$

\section{Numerical tools}

In the present study, sensitivities of integral parameters with respect to nuclear data are required. Those are calculated with a reactor physics code system CBZ[7], being developed at Hokkaido University. Multi-group cross section libraries CBZLIB are produced with the NJOY-99 code[8] and point-wise cross sections generated by the FRENDY code[9] from evaluated nuclear data libraries. Medium-dependent multi-group cross sections are calculated with CBZLIB based on JENDL-4.0[3], and these are used by the subsequent neutron transport calculations for a whole-system. Since all the neutron multiplicative systems considered in the present study have spherical geometry, the neutron transport equation is solved numerically by the SNR module of CBZ, which is based on discreteordinate angular discretization method for spherical systems. Forward and adjoint angular neutron fluxes are calculated with SNR, and sensitivities are calculated with the classical 
perturbation theory for effective neutron multiplication factor $k_{\text {eff }}$ and with the generalized perturbation theory for reaction rate ratio. In the following numerical results section, we will treat two problems: fictitious systems and actual systems. For calculations of the former, 276-energy group libraries are used. Energy division under the constant lethargy width of $1 / 16$ is adopted to an energy range from $10 \mathrm{MeV}$ to $0.3224 \mathrm{eV}$. For calculations of the latter, 70-energy group libraries are used. Energy division under the constant lethargy width of $1 / 4$ is adopted to an energy range from $10 \mathrm{MeV}$ to $0.3224 \mathrm{eV}$, and one more energy group from $0.3224 \mathrm{eV}$ to $10^{-5} \mathrm{eV}$ is added as the final group.

\section{Numerical results}

First the proposed methods are adopted to a set of fictitious integral data with a limited number of nuclear data to check its validity and to grasp its feature. After that, they are adopted to a set of actual integral data with a wide variety of nuclear data.

\subsection{Fictitious integral data}

Fictitious bare spherical critical assemblies made of homogeneous uranium metal with different U-235 enrichment are considered. A critical assembly with U-235 enrichment of $6 \mathrm{wt} \%$ is firstly considered, and other different assemblies are prepared by increasing U-235 enrichment by 1 wt\%. Finally 95 fictitious critical assemblies are prepared. It is assumed that the uranium metal fuel is made only of U-235 and -238. Radius of each of these critical assemblies is determined so as to make $k_{\text {eff }}$ of the assembly unity in numerical calculations with 276-group cross sections.

As a basis of nuclear data space, only U-235 fission cross sections are considered, so the number of integral data and nuclear data are 95 and 276 in the present case.

Sensitivities of $k_{\text {eff }}$ to 276 -group U-235 fission cross sections are shown in Figure 2 for some of the critical assemblies with different U-235 enrichments. Radius of the critical 
assemblies becomes small as U-235 enrichment of loaded fuel becomes large, so energy spectra of sensitivities become hard in the high-enriched uranium assemblies. Absolute values of sensitivities are relatively large in the high-enriched uranium assemblies since contribution of U-238 to total fission reaction rate becomes small.

[Figure 2 about here.]

The 276-group sensitivities are collapsed to 138-group and 69-group ones in order to see an effect of energy-group structure in the proposed method. These 138- and 69group structures are based on the equi-lethargy width for all the energy groups, and their lethargy widths are $1 / 8$ and $1 / 4$. Then sensitivity matrices are created from a set of sensitivity vectors based on 69, 138 and 276-group representations, and SVD is adopted to these matrices. Figure 3 shows singular values distributions of the sensitivity matrices. Singular values are normalized so as to make the first one unity. A rapid decrease is observed in the first ten singular values, and the decrease is moderated in higher-order singular values in every case. From mathematical aspect, singular values during the rapid decrease are non-negligible, and those larger than the tenth order can be regarded as noise components. However, a difference in singular values between the first one and the tenth one is quite significant, so it can be said from physical aspect that the tenth singular value component is negligible in comparison with the first one.

[Figure 3 about here.]

The dimension of the integral data space is determined by the proposed method using minimum norms of orthogonally-projected vectors. Norms of orthogonally-projected vectors of each normalized sensitivity vector are shown in Figure 4. Behavior of the norms are not dependent on the number of energy groups. As described in the preceding section, a criterion of the minimum norms should be determined, and it is strongly related to the accuracy of sensitivity vectors. Here we set a criterion as 0.99 , and it is equivalent to assume that accuracy of each entry of sensitivity vectors is around $1 \%$. With this criterion, 
the dimension of the integral data space considered here is determined as three while the total number of the integral data is 95 .

[Figure 4 about here.]

Figure 5 shows three basis vectors of the constructed integral data space. Shapes of energy profiles of the basis vectors are not dependent on the number of energy groups. Important integral data to construct the integral data space among 95 integral data are chosen by the proposed algorithm, and the critical assemblies with U-235 enrichments of $53 \mathrm{wt} \%, 6 \mathrm{wt} \%$ and $100 \mathrm{wt} \%$ are chosen. The criterion of the minimum norm is set as 0.99. This chosen set is reasonable since the first one has intermediate property of this set of integral data, and the second and third ones have extreme property among this integral data set.

[Figure 5 about here.]

Finally possibility of independent nuclear data validation from a set of these fictitious integral data is quantified with the proposed method. The result is shown in Figure 6 . To see an impact of criteria used in the integral data space dimension determination, calculation results with different number of dimensions are also presented. When the number of energy groups is decreased, the independent validation possibilities become large. Multi-group cross sections are energy-averaged cross sections, so if the energy range of averaging is wide, validation of the energy-averaged cross section should become easy. The present result suggests that independent validation of multi-group cross sections finer than 69-group from this set of integral data is difficult, and that independent validation of cross sections around several hundreds of $\mathrm{keV}$ and several $\mathrm{MeV}$ are relatively easy in comparison with cross sections in other energy ranges. Also it is interesting to point out that the validation possibility becomes large when the number of dimensions of the integral data space is large. Theoretically speaking, if sensitivity profiles can be calculated very accurately, criteria to determine subspace dimension can be loosened, and validation 
possibility is increased.

[Figure 6 about here.]

From the above results with the fictitious integral data, we can better understand the proposed concepts.

\subsection{Actual integral data}

The proposed method is adopted to a set of actual integral data shown in Table 1. All of these integral data are reactor physics parameters obtained at fast neutron systems, and the integral data with indices 1 to 14 are for $k_{\text {eff }}$, and the others are for fission reaction rate ratio at a core center position. The integral data whose ID are from 10 to 14 are presented in Table 1 with the specific terminology in ICSBEP, and its detail is omitted here. The terms F25, F28, F37 and F23 stand for fission reaction rates of U-235, -238, Np-237 and U-233, respectively. Sensitivities of $k_{\text {eff }}$ are relatively small in comparison with those of reaction rate ratio, so importance of the integral data about $k_{\text {eff }}$ becomes smaller than those about reaction rate ratio. In order to avoid this, all the sensitivities are normalized so as to make the Euclidean norm of each sensitivity unity. The user-defined criterion in our proposed methods is set 0.99 as in the preceding subsection.

[Table 1 about here.]

As nuclear data, we consider 8 reactions of fission, $(\mathrm{n}, \mathrm{f})$; neutron capture, $(\mathrm{n}, \gamma)$; elastic scattering, (n,n); inelastic scattering, $\left(\mathrm{n}, \mathrm{n}^{\prime}\right) ;(\mathrm{n}, 2 \mathrm{n})$; averaged cosine of elastic scattering angle, $\bar{\mu}$; averaged number of neutrons generated by one fission reaction, $\bar{\nu}$ and fission spectrum, $\chi$ for the following ten nuclides: U-233, -234, -235, -238, Pu-239, -240, -241, -242, Th-232 and Np-237. Corresponding indices of these nuclear data are listed in Table 2. Since the number of energy groups is 70 , the dimension of the nuclear data space considered here is $8 \times 10 \times 70=5,600$.

[Table 2 about here.] 
A normalized-singular values distribution of the sensitivity matrix is shown in Figure 7. The singular values distribution is different from those obtained from the fictitious integral data in the preceding subsection; noise components are not observed. A dimension of the integral data space spanned by these sensitivities is calculated as 13 with the procedure mentioned above. Since the total number of integral data is 32 , it can be concluded that the present data set includes highly-dependent integral data.

[Figure 7 about here.]

The principal three basis vectors having the largest singular values are shown in Figure 8. On the first basis vector, a large positive component is observed in $(n, f)$ cross sections of U-235 and negative components are observed in (n,f) cross sections of U-233, -238, Pu-239 and Np-237. These would be due to contribution of the integral data about fission reaction rate ratios in which all the denominators are the U-235 reaction rate. Among these negative components, those in (n,f) cross sections of U-238 and Np-237 are larger than the others since $(\mathrm{n}, \mathrm{f})$ cross sections of $\mathrm{U}-238$ and Np-237 have small or no contributions in the other integral data about $k_{\text {eff }}$ and are independent on $\bar{\nu}$ from the view point of sensitivity profiles. On the second basis vector, the first and second highest peaks are observed in $\bar{\nu}$ and $(\mathrm{n}, \mathrm{f})$ cross sections of U-233. This can be considered due to contribution of the integral data about $k_{\text {eff }}$ of the U-233-loaded cores to which both of $\bar{\nu}$ and $(\mathrm{n}, \mathrm{f})$ cross sections of U-233 are sensitive. On the third basis vector, the first and second highest peaks are observed in $\bar{\nu}$ and (n,f) cross sections of $\mathrm{Pu}-239$, and this would be due to contribution of the integral data about $k_{\text {eff }}$ of the $\mathrm{Pu}$-239-loaded cores. Note that the normalized singular values corresponding to these second and third basis vectors are close to each other: 0.80 and 0.73.

[Figure 8 about here.]

All the basis vectors corresponding to the U-235 (n,f) cross section are shown in Figure 9. Several basis vectors have non-negligible contributions to the U-235 (n,f) cross 
sections. This suggests that the basis vectors of the integral data space are quite complicated from a viewpoint of nuclear data.

[Figure 9 about here.]

To prepare a minimum set of the integral data, the following 18 integral data are chosen by the proposed method. These are presented in the descending order based on their priority: $32,1,11,8,25,21,4,28,19,23,5,26,27,30,7,22,9$ and 2. Detailed information on the first five chosen integral data are shown in Table 3. A wide variety of integral data is chosen by the proposed algorithm. The integral data about $k_{\text {eff }}$ of the $\mathrm{Pu}$-239-fueled core is chosen as the second priority although large contributions of $\bar{\nu}$ and $(\mathrm{n}, \mathrm{f})$ cross sections of $\mathrm{Pu}-239$ are found in not the second but the third basis vector. The reason why this integral data is chosen in prior to the integral data about $k_{\text {eff }}$ of the U-233-fueled core would be that the firstly-chosen integral data is the U-233-fueled core, and nuclear data such as (n,n') cross section of U-233 should be sensitive to this integral data as well as the thirdly-chosen integral data. Here let us remember that the second integral data is chosen with the proposed procedure because its sensitivity profile is the most different from the first one. Furthermore the singular values corresponding to these concerned basis vectors are close to each other as described above, so this disagreement between the order of the chosen integral data and that in the principal basis vectors of the integral data space is not a serious problem

[Table 3 about here.]

Finally independent validation possibility of nuclear data is quantified with the proposed method, and the result is shown in Figure 10. The $(n, f)$ cross sections of some nuclides show relatively high possibility in comparison with others, but it is smaller than 0.5. Independent validation possibility of $(\mathrm{n}, \mathrm{f})$ cross sections is shown in Figure 11.

[Figure 10 about here.]

[Figure 11 about here.] 


\subsection{On the nuclear data compensation effect}

The proposed concept based on the active subspace is used to interpret the compensation effect in evaluated nuclear data libraries.

The nuclear data compensation effect is that recent evaluated nuclear data libraries predict quite well some integral data although there exist significant differences in nuclear data among these nuclear data libraries. Figure 12 shows $\mathrm{C} / \mathrm{E}$ values of $k_{\text {eff }}$ of 14 fast neutron systems, which are treated in the present study. The recent evaluated nuclear data libraries, JENDL-4.0, ENDF/B-VII.1[10] and JEFF-3.2[11], predict experimental values quite well. On the other hand, a relatively old evaluated nuclear data library, JENDL3.2[12], shows much worse performance than the other three libraries.

[Figure 12 about here.]

Figure 13 shows (n,n') cross sections of Pu-239 taken from these evaluated nuclear data libraries. This cross section significantly affects $k_{\text {eff }}$ concerned here[13]. Evaluated cross section data are different from each other, and a difference among the three recent data libraries is large. These differences result in differences in calculated $k_{\text {eff }}$, but differences in other nuclear data cancel this effect, and finally predicted $k_{\text {eff }}$ becomes almost same among the different nuclear data libraries. This is an example of the nuclear data compensation effect.

[Figure 13 about here.]

Even though nuclear data evaluations are significantly different from each other, if the compensation effect is observed, this means that nuclear data evaluations among different libraries should be almost same in the integral data space. In order to confirm this, nuclear data in the integral data space are calculated for these libraries. Here the integral data space is constructed from 14 integral data related to $k_{\text {eff }}$ since the compensation effect is not observed in the integral data about the reaction rate ratio. The dimension of this integral data space is calculated as 5 , but in this calculation, a 14-dimensional 
space is considered. Since sensitivities are defined as relative quantities, nuclear data in this integral data space should be defined also as relative quantities. Here the JENDL4.0 evaluation is considered as a reference, and a relative difference to this reference is regraded as nuclear data. The nuclear data represented in this integral data space are calculated as shown in Figure 14. As expected, differences of the recent data libraries ENDF/B-VII.1 and JEFF-3.2 to the reference are very small in each basis vector of the integral data space while only the JENDL-3.2 evaluation shows a large difference. This is one interpretation of the nuclear data compensation effect.

[Figure 14 about here.]

\section{Conclusion}

To know performance of evaluated nuclear data in reactor physics or radiation shielding calculations, its benchmark testing with integral data is mandatory. Nowadays we have a huge amount of integral data, but some of them are quite similar with each other. We need to know independency of available integral data, and also to choose a proper set of a limited number of integral data for benchmark calculations. Furthermore, it is beneficial to know how effective a set of integral data is for independent validation of each nuclear data in performing validation test of nuclear data against integral data. In order to quantify the effectiveness of integral data in nuclear data validation, we have proposed several methods based on a concept of the active subspace. With the proposed methods, we can quantify independency of a set of integral data, choose a minimum set of proper integral data, and quantify possibility of independent validation of nuclear data from a set of integral data. These methods have been adopted to a set of fictitious integral data and a set of actual integral data including experimental data about $k_{\text {eff }}$ and reaction rate ratios. Through these applications, effectiveness of these integral data has been successfully quantified.

Furthermore, the proposed concept has been utilized to interpret the nuclear data 
compensation effect, which has been recently discussed in the community of nuclear data.

In the present study, we have used 32 actual integral data which are all fast neutron systems, and have considered nuclear data for 10 important actinoid nuclides. The considered integral data is just a fraction of integral data available for us at present, and the concerned energy range is also limited to a fast neutron range. Nuclear data of other nuclides such as light nuclei and medium-heavy nuclei are also important, and nuclear data other than reaction cross sections, such as fission yields and decay property, are also important in many aspects. Our final goal is to use all the available integral data and all the relevant nuclear data in application of the proposed methods.

\section{Acknowledgement}

The authors greatly appreciate for reviewers for their valuable comments.

\section{References}

[1] Briggs JB. Ed. International Handbook of Evaluated Critical Safety Benchmark Experiments. France: OECD, Nuclear Energy Agency; 2010, NEA/NSC/DOC(95)03.

[2] Mosteller RD. ENDF/B-VII.0, ENDF/B-VI, JEFF-3.1, and JENDL-3.3 results for unreflected plutonium solutions and MOX lattices. Proc. Joint Int. Meeting on Mathematics \& Computation and Supercomputing in Nuclear Applications; 2006 April 15-19; Monterey, California.

[3] Shibata K, Iwamoto O, Nakagawa T, et al. JENDL-4.0: A new library for nuclear science and technology. J Nucl Sci Technol. 2011; 48:1-30.

[4] Chiba G, Okumura K, Sugino K, Nagaya Y, Yokoyama K, Kogo T, Ishikawa M, Okajima S. JENDL4.0 benchmarking for fission reactor applications. J Nucl Sci Technol. 2011; 48:172-187.

[5] Abdel-Khalik HS, Turinsky PJ, Jessee MA. Efficient subspace methods-based algorithms for performing sensitivity, uncertainty, and adaptive simulation of large-scale computational models. Nucl Sci Eng. 2008; 159:256-272. 
[6] Palmiotti G, Salvatores M. Cross section covariances: a user perspective. EPJ Nucl Sci Technol. 2018; $4: 40$.

[7] Chiba G, Endo T. Numerical benchmark problem of solid-moderated enriched-U-loaded core at Kyoto university critical assembly. J Nucl Sci Technol. 2019; 57:187-195.

[8] MacFarlane RE. NJOY99.0: code system for producing pointwise and multigroup neutron and photon cross sections from ENDF/B data. Los Alamos National Laboratory; 1999.

[9] Tada K, Nagaya Y, Kunieda S, Suyama K, Fukahori T. Development and verification of a new nuclear data processing system FRENDY. J Nucl Sci Technol. 2017; 54:806-817.

[10] Chadwick MB, Herman M, Oblozinsky P, et al. ENDF/B-VII.1 Nuclear data for science and technology: cross sections, covariances, fission product yields and decay data. Nucl Data Sheets. 2011; 112:2887-2996.

[11] OECD Nuclear Energy Agency. JEFF-3.2 evaluated data library. https://www.oecd-nea.org/dbforms/data/eva/evatapes/jeff_32/. 2014;

[12] Nakagata T, Shibata K, Chiba S, et al. Japanese evaluated nuclear data library version 3 revision-2: JENDL-3.2. J Nucl Sci Technol. 1995; 32:1259-1271.

[13] Plompen A, Kawano T, Capote RN. Summary report of technical meeting on inelastic scattering and capture cross-section data of major actinoids in the fast neutron range. INDC(NDS)-0597; International Atomic Energy Agency; 2012. 
Table 1 Used actual integral data with their indices

\begin{tabular}{|c|c||c|c|}
\hline Index & Name & Index & Name \\
\hline 1 & Jezebel & 17 & F23/F25 in Godiva \\
2 & Jezebel-Pu & 18 & F49/F25 in Godiva \\
3 & Jezebel-233 & 19 & F28/F25 in Jezebel \\
4 & Godiva & 20 & F37/F25 in Jezebel \\
5 & Flattop-Pu & 21 & F23/F25 in Jezebel \\
6 & Flattop-U & F49/F25 in Jezebel \\
7 & Flattop-233 & 23 & F28/F25 in Jezebel-233 \\
8 & Big-ten & 24 & F37/F25 in Jezebel-233 \\
9 & Thor (Pu w Th Ref.) & 26 & F37/F25 in Flattop-U \\
10 & PMF010 (Pu w NU Ref.) & 27 & F23/F25 in Flattop-U \\
11 & U3MF002-1 (U-233 w HEU Ref.) & 28 & F49/F25 in Flattop-U \\
12 & U3MF002-2 (U-233 w HEU Ref.) & 28 in Flattop-U \\
13 & U3MF003-1 (U-233 w NU Ref.) & 29 & F28/F25 in Flattop-Pu \\
14 & U3MF003-2 (U-233 w NU Ref.) & 30 & F37/F25 in Flattop-Pu \\
15 & F28/F25 in Godiva & 31 & F28/F25 in Flattop-233 \\
16 & F37/F25 in Godiva & 32 & F37/F25 in Flattop-233 \\
\hline
\end{tabular}


Table 2 Nuclear data with their index.

\begin{tabular}{|c|c|c|c|c|c|c|c|c|}
\hline Index & Nuclide & ND & Index & Nuclide & ND & Index & Nuclide & ND \\
\hline $1-$ & U-233 & $(\mathrm{n}, \mathrm{f})$ & 2241- & $\mathrm{Pu}-239$ & $(n, f)$ & 4481- & Th-232 & $(n, f)$ \\
\hline 71- & U-233 & $(\mathrm{n}, \gamma)$ & 2311- & $\mathrm{Pu}-239$ & $(\mathrm{n}, \gamma)$ & $4551-$ & Th-232 & $(\mathrm{n}, \gamma)$ \\
\hline 141- & $\mathrm{U}-233$ & $(\mathrm{n}, \mathrm{n})$ & 2381- & Pu-239 & $(\mathrm{n}, \mathrm{n})$ & 4621- & Th-232 & $(n, n)$ \\
\hline 211- & $\mathrm{U}-233$ & $\left(\mathrm{n}, \mathrm{n}^{\prime}\right)$ & 2451- & $\mathrm{Pu}-239$ & $\left(n, n^{\prime}\right)$ & 4691- & Th-232 & $\left(\mathrm{n}, \mathrm{n}^{\prime}\right)$ \\
\hline 281- & $\mathrm{U}-233$ & $(\mathrm{n}, 2 \mathrm{n})$ & $2521-$ & $\mathrm{Pu}-239$ & $(\mathrm{n}, 2 \mathrm{n})$ & 4761- & Th-232 & $(\mathrm{n}, 2 \mathrm{n})$ \\
\hline $351-$ & U-233 & $\bar{\mu}$ & 2591- & $\mathrm{Pu}-239$ & $\bar{\mu}$ & 4831- & Th-232 & $\bar{\mu}$ \\
\hline 421- & $\mathrm{U}-233$ & $\bar{\nu}$ & 2661- & $\mathrm{Pu}-239$ & $\bar{\nu}$ & 4901- & Th-232 & $\bar{\nu}$ \\
\hline 491- & U-233 & $\chi$ & 2731- & $\mathrm{Pu}-239$ & $\chi$ & 4971- & Th-232 & $\chi$ \\
\hline 561- & $\mathrm{U}-234$ & $(\mathrm{n}, \mathrm{f})$ & 2801- & $\mathrm{Pu}-240$ & $(\mathrm{n}, \mathrm{f})$ & $5041-$ & Np-237 & $(n, f)$ \\
\hline 631- & U-234 & $(\mathrm{n}, \gamma)$ & 2871- & $\mathrm{Pu}-240$ & $(\mathrm{n}, \gamma)$ & 5111- & Np-237 & $(n, \gamma)$ \\
\hline 701- & U-234 & $(\mathrm{n}, \mathrm{n})$ & 2941- & $\mathrm{Pu}-240$ & $(\mathrm{n}, \mathrm{n})$ & 5181- & Np-237 & $(n, n)$ \\
\hline 771- & $\mathrm{U}-234$ & $\left(\mathrm{n}, \mathrm{n}^{\prime}\right)$ & 3011- & $\mathrm{Pu}-240$ & $\left(n, n^{\prime}\right)$ & $5251-$ & Np-237 & $\left(\mathrm{n}, \mathrm{n}^{\prime}\right)$ \\
\hline 841- & $\mathrm{U}-234$ & $(\mathrm{n}, 2 \mathrm{n})$ & 3081- & $\mathrm{Pu}-240$ & $(\mathrm{n}, 2 \mathrm{n})$ & $5321-$ & Np-237 & $(\mathrm{n}, 2 \mathrm{n})$ \\
\hline 911- & U-234 & $\bar{\mu}$ & $3151-$ & $\mathrm{Pu}-240$ & $\bar{\mu}$ & 5391- & Np-237 & $\bar{\mu}$ \\
\hline 981- & U-234 & $\bar{\nu}$ & $3221-$ & $\mathrm{Pu}-240$ & $\bar{\nu}$ & 5461- & Np-237 & $\bar{\nu}$ \\
\hline $1051-$ & U-234 & $\chi$ & 3291- & $\mathrm{Pu}-240$ & $\chi$ & 5531- & Np-237 & $\chi$ \\
\hline 1121- & $\mathrm{U}-235$ & $(\mathrm{n}, \mathrm{f})$ & 3361- & $\mathrm{Pu}-241$ & $(n, f)$ & & & \\
\hline 1191- & U-235 & $(\mathrm{n}, \gamma)$ & $3431-$ & $\mathrm{Pu}-241$ & $(\mathrm{n}, \gamma)$ & & & \\
\hline $1261-$ & $\mathrm{U}-235$ & $(\mathrm{n}, \mathrm{n})$ & $3501-$ & $\mathrm{Pu}-241$ & $(\mathrm{n}, \mathrm{n})$ & & & \\
\hline 1331- & U-235 & $\left(\mathrm{n}, \mathrm{n}^{\prime}\right)$ & $3571-$ & $\mathrm{Pu}-241$ & $\left(n, n^{\prime}\right)$ & & & \\
\hline $1401-$ & $\mathrm{U}-235$ & $(\mathrm{n}, 2 \mathrm{n})$ & $3641-$ & $\mathrm{Pu}-241$ & $(\mathrm{n}, 2 \mathrm{n})$ & & & \\
\hline 1471- & U-235 & $\bar{\mu}$ & $3711-$ & $\mathrm{Pu}-241$ & $\bar{\mu}$ & & & \\
\hline $1541-$ & $\mathrm{U}-235$ & $\bar{\nu}$ & $3781-$ & $\mathrm{Pu}-241$ & $\bar{\nu}$ & & & \\
\hline 1611- & U-235 & $\chi$ & $3851-$ & $\mathrm{Pu}-241$ & $\chi$ & & & \\
\hline 1681- & U-238 & $(\mathrm{n}, \mathrm{f})$ & $3921-$ & $\mathrm{Pu}-242$ & $(\mathrm{n}, \mathrm{f})$ & & & \\
\hline 1751- & U-238 & $(\mathrm{n}, \gamma)$ & 3991- & $\mathrm{Pu}-242$ & $(\mathrm{n}, \gamma)$ & & & \\
\hline 1821- & U-238 & $(\mathrm{n}, \mathrm{n})$ & 4061- & $\mathrm{Pu}-242$ & $(\mathrm{n}, \mathrm{n})$ & & & \\
\hline 1891- & U-238 & $\left(\mathrm{n}, \mathrm{n}^{\prime}\right)$ & 4131- & $\mathrm{Pu}-242$ & $\left(n, n^{\prime}\right)$ & & & \\
\hline 1961- & U-238 & $(\mathrm{n}, 2 \mathrm{n})$ & $4201-$ & $\mathrm{Pu}-242$ & $(\mathrm{n}, 2 \mathrm{n})$ & & & \\
\hline 2031- & U-238 & $\bar{\mu}$ & $4271-$ & $\mathrm{Pu}-242$ & $\bar{\mu}$ & & & \\
\hline 2101- & U-238 & $\bar{\nu}$ & $4231-$ & $\mathrm{Pu}-242$ & $\bar{\nu}$ & & & \\
\hline 2171- & $\mathrm{U}-238$ & $\chi$ & 4411- & $\mathrm{Pu}-242$ & $\chi$ & & & \\
\hline
\end{tabular}


Table 3 Detailed information on the chosen integral data

\begin{tabular}{|c|c|c|c|c|}
\hline Priority & Index & Parameter & Fuel material & Reflector material \\
\hline 1 & 32 & F37/F25 & U-233 & U-238 \\
2 & 1 & $k_{\text {eff }}$ & Pu-239 & - \\
3 & 11 & $k_{\text {eff }}$ & U-233 & U-235 \\
4 & 8 & $k_{\text {eff }}$ & U-235, -238 & U-238 \\
5 & 25 & F28/F25 & U-235 & U-238 \\
\hline
\end{tabular}




\section{Figure Captions}

Figure 1 Relation of an integral data space and a nuclear data vector

Figure 2 Sensitivity of $k_{\text {eff }}$ to U-235 fission cross sections

Figure 3 Singular values distributions of sensitivity matrices of fictitious critical assemblies with different energy group representations

Figure 4 Norms of orthgonally-projected vectors of each normalized sensitivity vector

Figure 5 Principal basis vectors spanning the integral data space of fictitious integral data

Figure 6 Possibility of independent nuclear data validation from a set of fictitious integral data

Figure 7 Singular values distribution of a sensitivity matrix consisting of 32 actual integral data

Figure 8 Principal basis vectors spanning the integral data space of 32 actual integral data

Figure 9 Basis vectors spanning the integral data space of 32 actual integral data $((n, f)$ cross section of $\mathrm{U}-235)$

Figure 10 Possibility of independent nuclear data validation from a set of actual integral data 
Figure 11 Possibility of independent nuclear data validation of (n,f) cross sections from a set of actual integral data

Figure $12 \mathrm{C} / \mathrm{E}$ values of the integral data about $k_{\text {eff }}$ obtained with several evaluated nuclear data libraries

Figure 13 Inelastic scattering cross section of $\mathrm{Pu}-239$

Figure 14 Relative difference of nuclear data defined in the integral data space (reference: JENDL-4.0) 
J. Nucl. Sci. \& Technol.

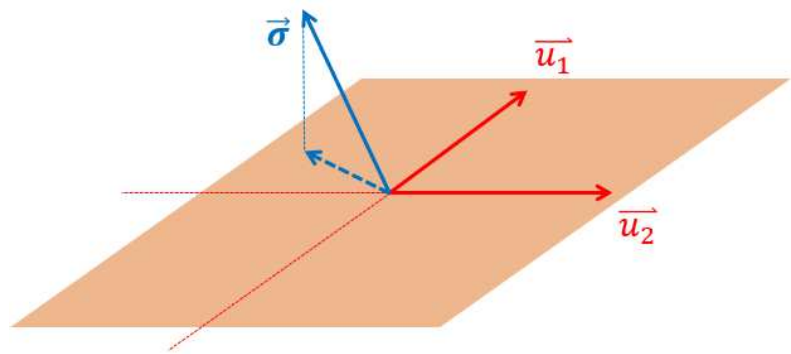

Figure 1 Relation of an integral data space and a nuclear data vector 
J. Nucl. Sci. \& Technol.

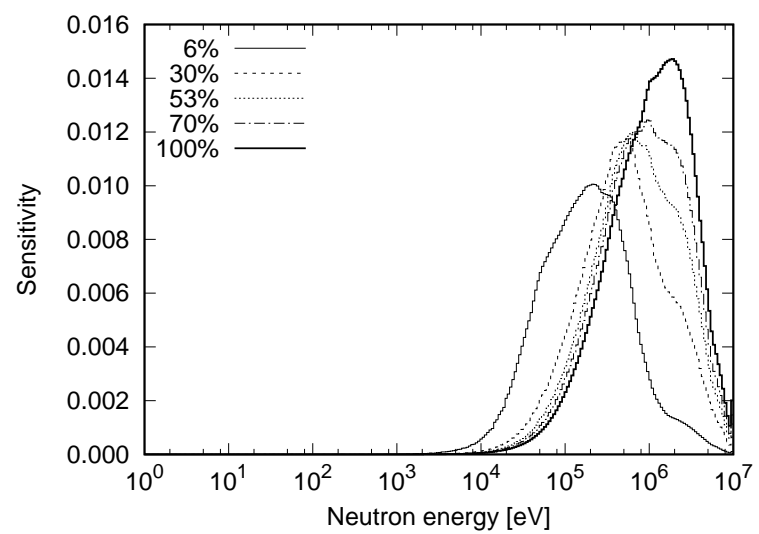

Figure 2 Sensitivity of $k_{\text {eff }}$ to U-235 fission cross sections 
J. Nucl. Sci. \& Technol.

Article

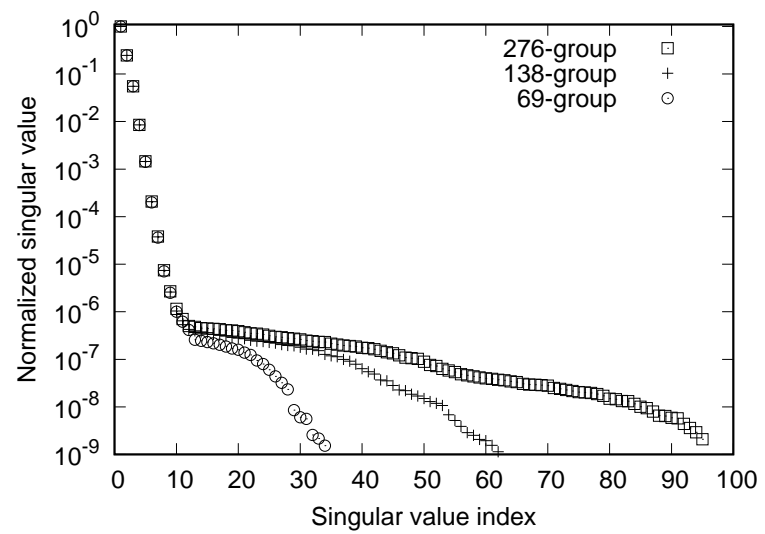

Figure 3 Singular values distributions of sensitivity matrices of fictitious critical assemblies with different energy group representations 
(a) 276-group

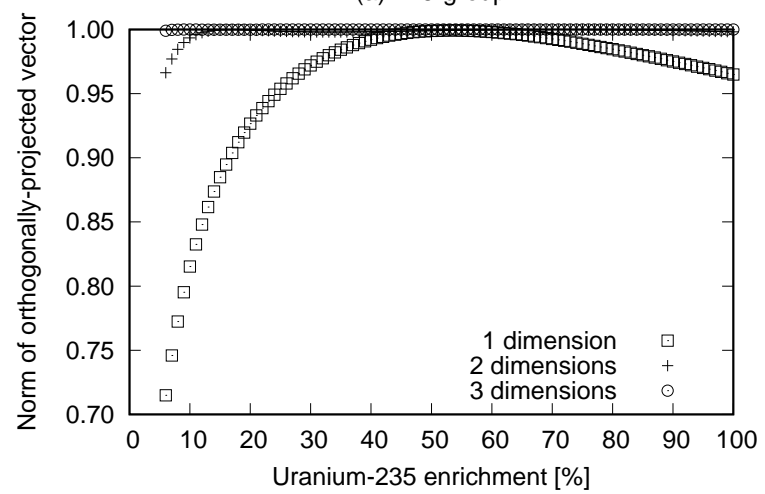

(a) 69-group

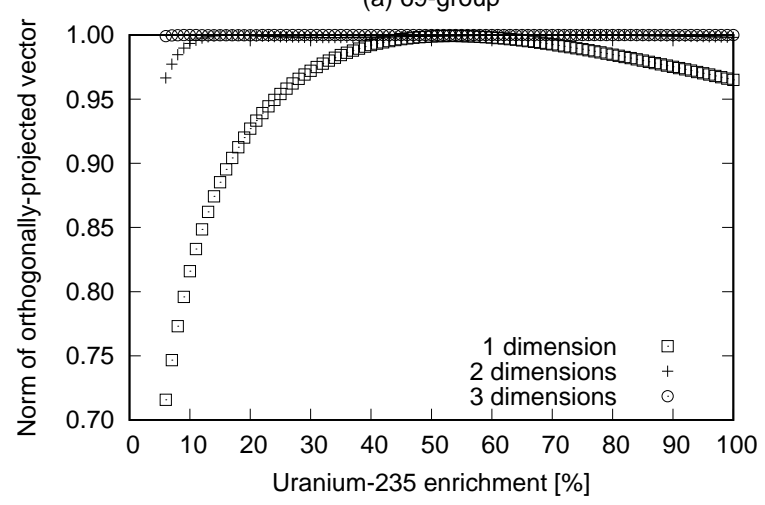

(b) 138-group

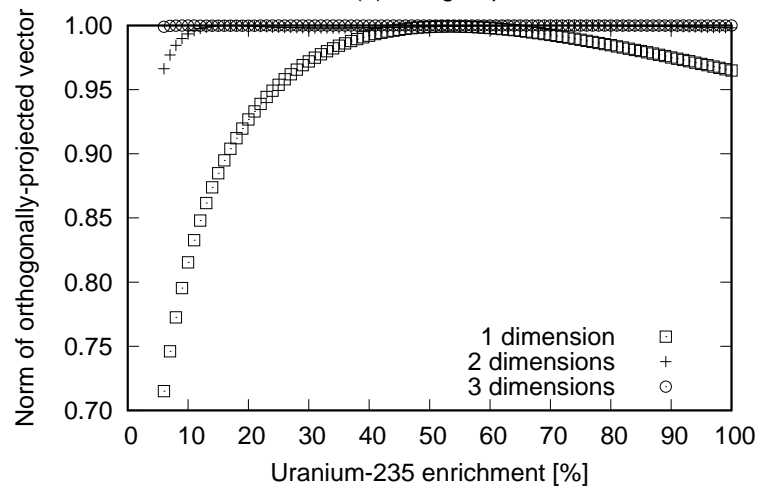

Figure 4 Norms of orthgonally-projected vectors of each normalized sensitivity vector 
(a) 276-group

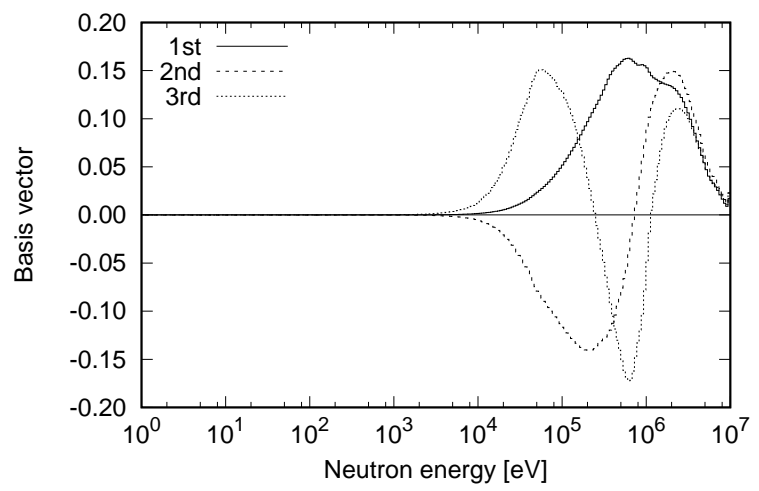

(c) 69-group

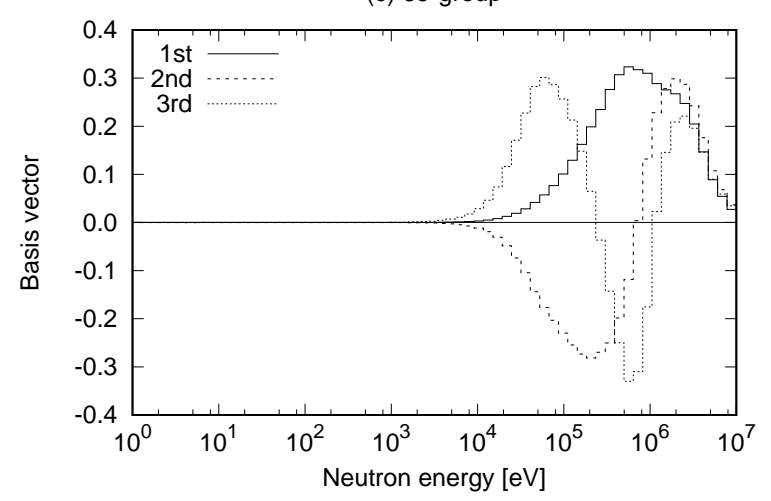

(b) 138-group

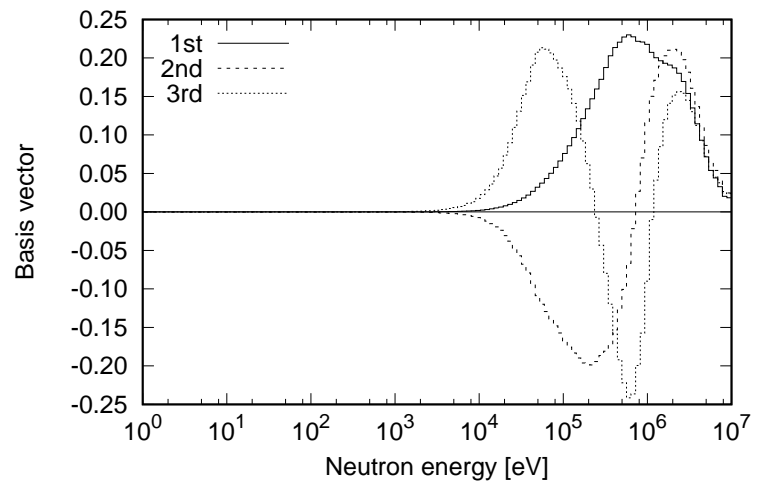

Figure 5 Principal basis vectors spanning the integral data space of fictitious integral data 
(a) 3-dimension

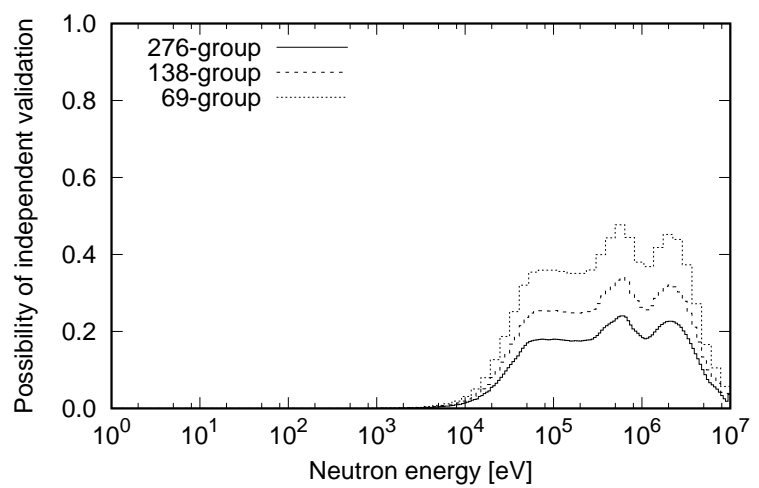

(b) 5-dimension

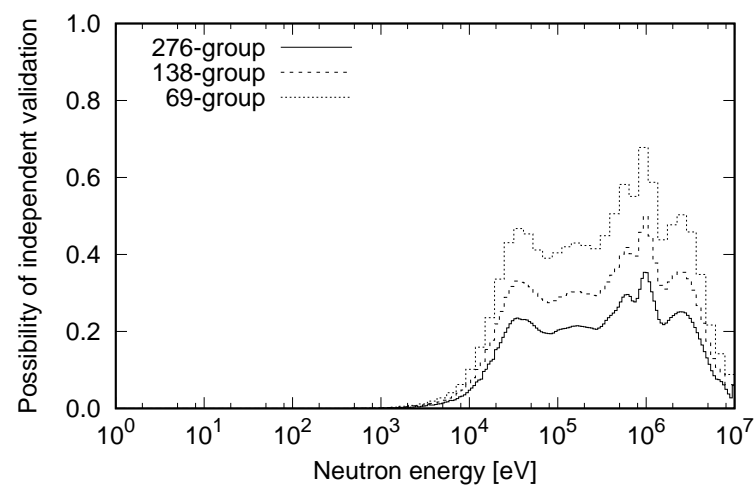

Figure 6 Possibility of independent nuclear data validation from a set of fictitious integral data 
J. Nucl. Sci. \& Technol.

Article

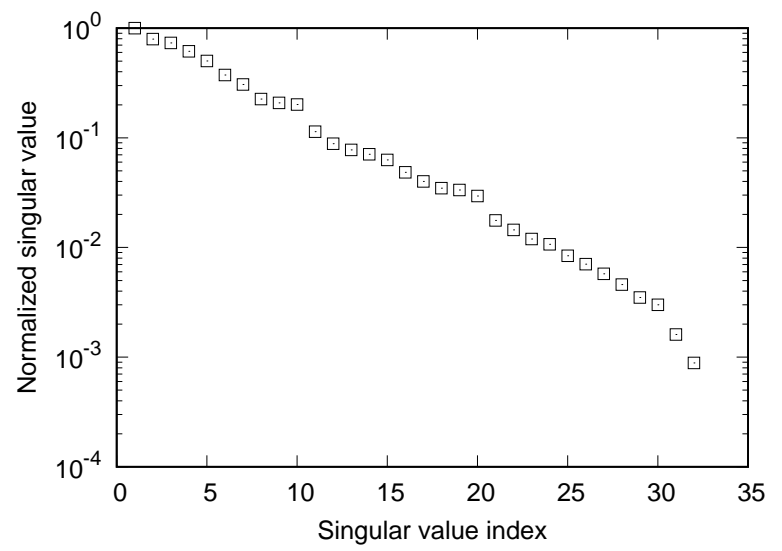

Figure 7 Singular values distribution of a sensitivity matrix consisting of 32 actual integral data 

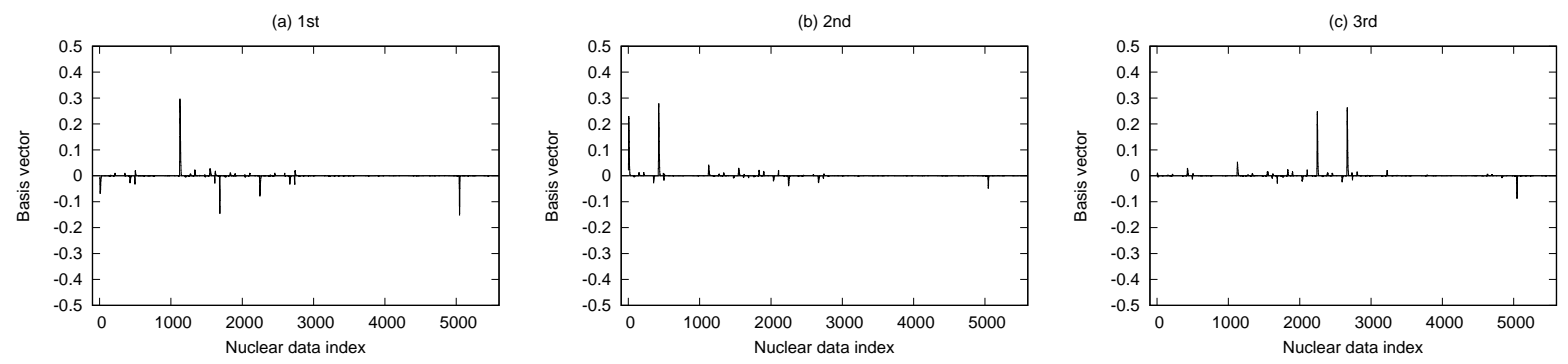

Figure 8 Principal basis vectors spanning the integral data space of 32 actual integral data 

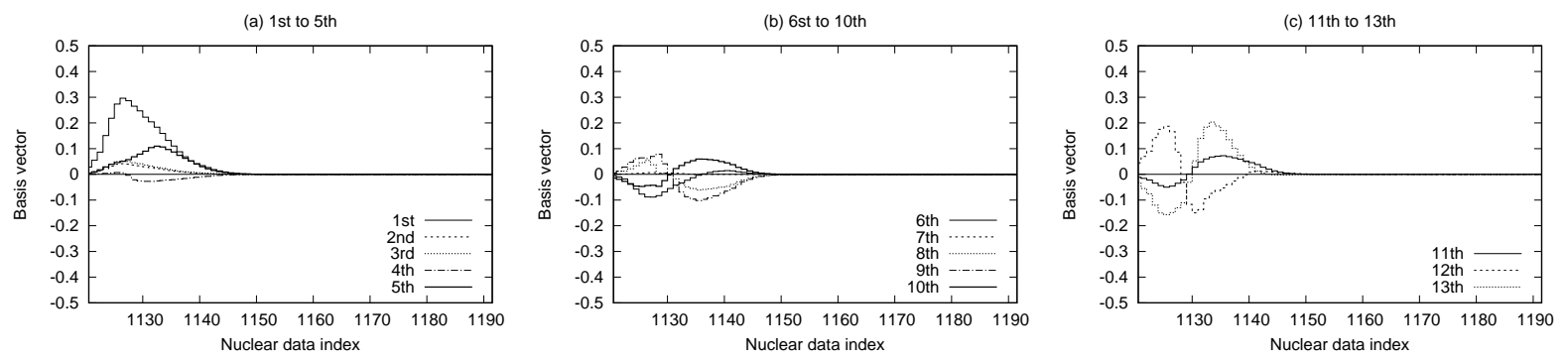

Figure 9 Basis vectors spanning the integral data space of 32 actual integral data ((n,f) cross section of U-235) 
J. Nucl. Sci. \& Technol.

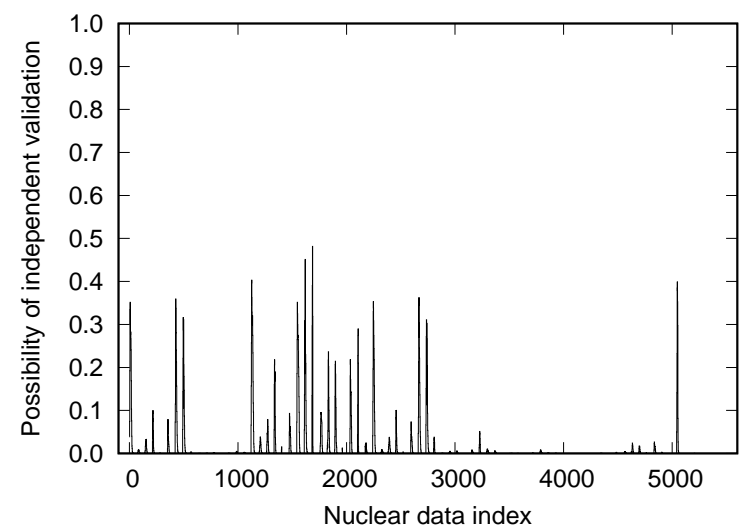

Figure 10 Possibility of independent nuclear data validation from a set of actual integral data 
J. Nucl. Sci. \& Technol.

Article

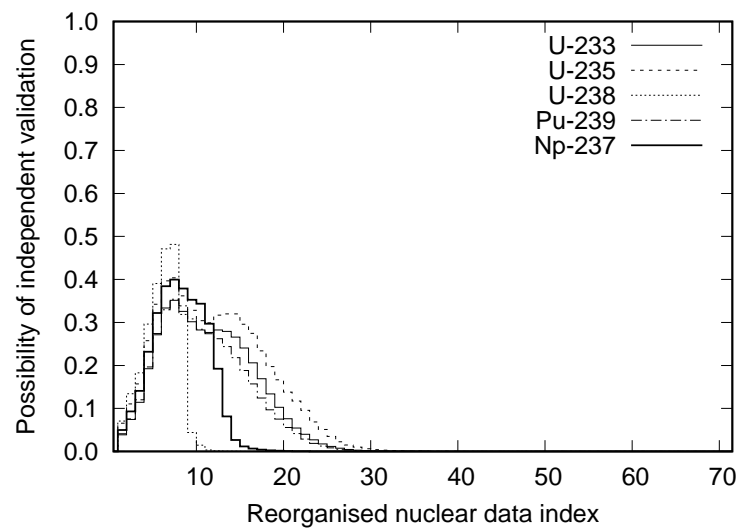

Figure 11 Possibility of independent nuclear data validation of $(\mathrm{n}, \mathrm{f})$ cross sections from a set of actual integral data 
J. Nucl. Sci. \& Technol.

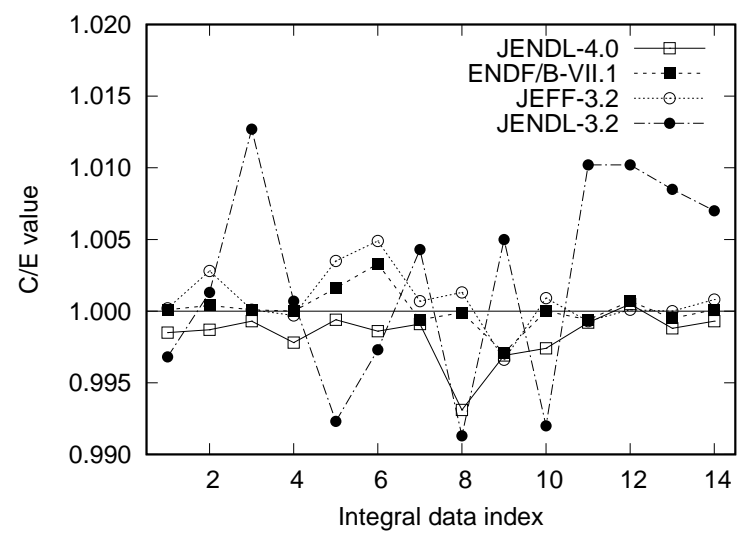

Figure $12 \mathrm{C} / \mathrm{E}$ values of the integral data about $k_{\text {eff }}$ obtained with several evaluated nuclear data libraries 
J. Nucl. Sci. \& Technol.

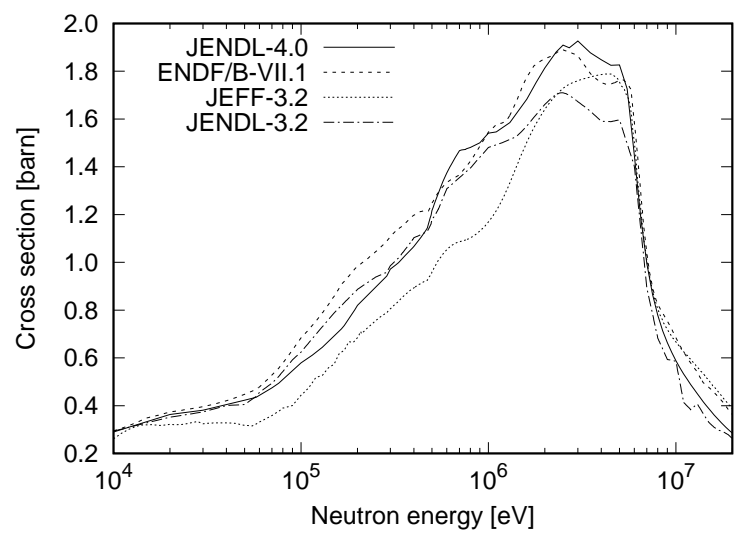

Figure 13 Inelastic scattering cross section of $\mathrm{Pu}-239$ 
J. Nucl. Sci. \& Technol.

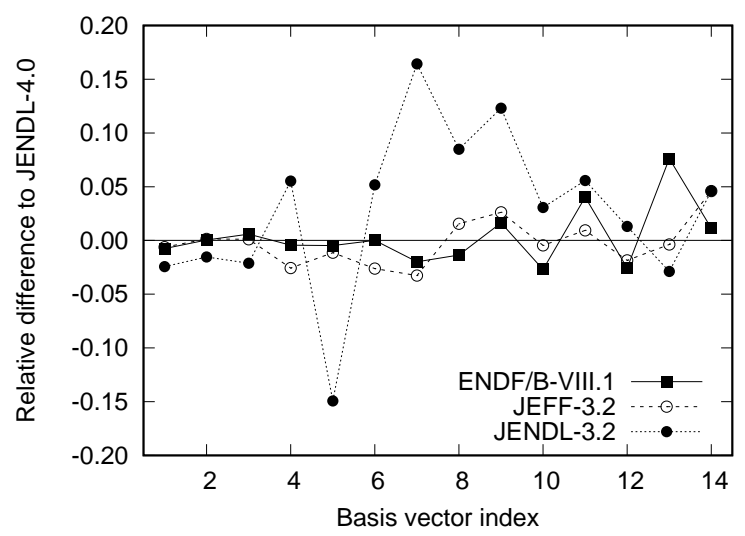

Figure 14 Relative difference of nuclear data defined in the integral data space (reference: JENDL-4.0) 